\title{
«Las voces infantiles llegan a ser tan potentes que es imposible no escucharlas»: entrevista a Susana Sosenski ${ }^{1}$
}

\author{
Juan Carlos Amador Baquiro²
}

\section{Presentación 3}

Susana Sosenski es licenciada en Estudios Latinoamericanos por la Universidad Nacional Autónoma de México (Unam) y doctora en historia por El Colegio de México. Adelantó una estancia posdoctoral en el Instituto de Investigaciones Sociales de la Unam. Desde 2010 está vinculada al Instituto de Investigaciones Históricas de esta misma universidad y está adscrita al Sistema Nacional de Investigadores en el Programa de Primas al Desempeño del Personal Académico de Tiempo Completo de México.

La doctora Sosenski ha sido pionera en indagar la presencia de los niños y las niñas en la historia de México y América Latina y el Caribe. Si bien existen abundantes trabajos historiográficos de la infancia en la región, especialmente construidos desde el análisis crítico de los discursos de la protección y las políticas de representación sobre la infancia y la familia, las investigaciones de Susana Sosenski auscultan distintas formas de participación infantil en la historia y develan la capacidad de agencia y resistencia de estos actores sociales en medio de

\footnotetext{
${ }^{1}$ Investigadora del Instituto de Investigaciones Históricas de la Universidad Nacional Autónoma de México.

2 Profesor titular Universidad Distrital Francisco José de Caldas. Profesor catedrático del Doctorado en Ciencias Sociales, Niñez y Juventud (Universidad de Manizales-Cinde).

3 Algunos de estos datos fueron tomados con ajustes del sitio web de la Universidad Nacional Autónoma de México (Instituto de Investigaciones Históricas): http://www.historicas.unam.mx/investigacion/investigadores/ sosenski.html
} 
condiciones adversas, intervenidas casi siempre por la experiencia colonial y sus respectivas herencias: adultocentrismo, clasismo, patriarcado y racismo.

Una de las contribuciones más importantes de la doctora Sosenski es la exploración de la historia de la infancia en México, desde ámbitos polémicos e incómodos para el mundo adulto. En primer lugar, ha analizado la presencia de los niños y las niñas en el sistema económico (en los siglos XIX y XX) a partir de categorías complejas como trabajo infantil y consumo infantil. En segundo lugar, ha abordado la participación de los niños y las niñas en la guerra y en el exilio, así como la percepción de estos sobre diversos conflictos bélicos. Por último, y de manera más reciente, se ha ocupado de problematizar el consumo infantil y familiar en la historia reciente de México.

En su oficio de historiadora, uno de los aspectos más fascinantes de sus trabajos de investigación es la versatilidad con la que aborda diversos archivos y fuentes de información. Particularmente, se destacan fuentes como documentos judiciales y hemerográficos, materiales filmográficos, caricaturas, testimonios, diarios infantiles y material publicitario. Se trata de modos particulares de develar «las voces» de los niños y niñas en el contexto de hechos sociales, políticos y culturales (tal como lo sostiene en una de sus respuestas, la cual inspiró el título de la presente entrevista). Este modo de hacer historia de la infancia en México y la región ha hecho posible que su trabajo se haya difundido significativamente en América Latina y el Caribe, Estados Unidos y Europa, y la haya convertido en un referente no solo para la historia social y cultural, sino también para las ciencias sociales y los estudios sociales de infancia.

Su trayectoria la ha hecho merecedora del Premio a la Mejor Tesis de Doctorado en Ciencias Sociales y Humanidades, otorgado por la Academia Mexicana de Ciencias, y al Premio al Mejor Artículo del Periodo Siglo XX, otorgado por el Comité Mexicano de Ciencias Históricas (2006), el cual también le entregó una mención honorífica en el año 2010. En su obra se destacan más de zo trabajos académicos, entre artículos en revistas arbitradas, capítulos de libros y ponencias en memorias de congresos. Algunos de sus libros más destacados son Niños en acción: el trabajo infantil en la ciudad de México (1920-1934) (El Colegio de México, 2010), Nuevas miradas a la historia de la infancia en América Latina: entre prácticas y representaciones (coordinación editorial con Elena Jackson Albarrán, Universidad Nacional Autónoma de México, Instituto de Investigaciones Históricas, 2012), Cosa de todos los días: historia de la vida cotidiana en México, v. I, México prehispánico (Coordinación editorial con Estela Roselló Soberón y Valeria Sánchez Michel, SM Ediciones, 2014) y Cosa de todos los días: historia de la vida cotidiana en México, v. II, México colonial (con los mismos editores, SM Ediciones, 2015). 
Con esta presentación, me permito agradecer a la doctora Sosenski por conceder de manera generosa y cálida esta entrevista, así como por compartir parte de su trayectoria y perspectivas en este diálogo. Para la Revista Latinoamericana de Ciencias Sociales, Niñez y Juventud ${ }^{4}$ es un privilegio contar en esta edición con este testimonio, el cual devela no solo una postura epistémica, ontológica y política en torno a la historia de la infancia y los estudios sociales de infancia, sino también facetas biográficas de la doctora Susana que permiten entender su compromiso con la producción de conocimiento sobre esas otras infancias que históricamente se ubican en los bordes y los intersticios del poder, la hegemonía y la exclusión en América Latina y el Caribe.

Juan Carlos Amador Baquiro (J. A.): ¿Qué motivaciones personales, profesionales o intelectuales contribuyeron a interesarte por los estudios historiográficos de las infancias en México y América Latina y el Caribe?

Susana Sosenski (S. S.): La primera vez que comencé a interesarme por la historia de las infancias fue durante mis estudios de doctorado en historia en El Colegio de México. En mis cursos las preguntas que tenía en un principio - sobre el artesanado urbano en el siglo XIXterminaron enmarcándose en otros trabajadores urbanos del siglo XX y en menores de edad. Me gusta pensar también que esta decisión académica — que me ha llevado a estudiar a los niños del pasado- se relaciona con mi niñez. Siendo niña mis padres me llevaban a conocer distintos pueblos y ciudades de México. Les preocupaba que mi hermana y yo reconociéramos las diferencias entre nuestra infancia y las otras infancias mexicanas con las que nos íbamos encontrando. Es por eso que en nuestros álbumes familiares de aquella época, entre las fotos de los viajes, de mis cumpleaños, de mis abuelos o de las casas en donde viví, aparecen también las fotos que tomaba mi padre de esas otras infancias: niños trabajando en gasolineras, vendiendo juguetes de madera o haciendo trabajos en el campo. Sin duda, estas son solo piezas sueltas de un rompecabezas más grande; pero considero que el ejercicio de pensar por qué se escribe lo que se escribe, o cuál es nuestro lugar de enunciación, debería hacernos reconocer el peso que han tenido esas pequeñas piezas en ciertos momentos de nuestra vida.

Mi primer interés como investigadora me llevó a estudiar la historia del trabajo infantil en México y a entender cómo la sociedad mexicana había tratado el tema. Me enfoqué en demostrar, no solo que los niños y las niñas trabajadores debían tener un lugar en la historiografía,

4 La doctora Sosenski es integrante de nuestro Comité Científico; además, algunos de sus artículos han sido publicados en la revista y ha hecho parte del grupo de pares evaluadores previamente. 
sino que en ese lugar debíamos reconocerlos como protagonistas, como sujetos activos, partícipes; como agentes sociales que habían tenido la capacidad de transformar su realidad, de modificar su medio, de negociar con sus pares y construir sociabilidades, aún a pesar de los esfuerzos, siempre presentes, siempre violentos, de una sociedad adultocéntrica que pretendía limitar la autonomía infantil. Estas ideas quedaron plasmadas en Niños en acción: el trabajo infantil en la ciudad de México (1920-1934).

Por otras piezas del rompecabezas de mi historia personal, hace ya muchos años que mi quehacer académico ha estado marcado por una suerte de pulsión bolivariana, que me empuja constantemente a buscar tejer vínculos y redes entre quienes nos interesamos por las infancias latinoamericanas. Aunque mis estudios sobre infancias se han concentrado en el caso mexicano, me ha interesado buscar cuáles son los puntos en común y las diferencias que comparten los casos nacionales en Latinoamérica. Así coordiné, con otras colegas, libros colectivos como Nuevas Miradas a la infancia: la historia de la infancia en América Latina, o más recientemente Violencia e infancias en el cine latinoamericano. Co-coordiné también dossiers como «Espacios y cultura material para la infancia en América Latina (siglos XIX y XX)» en la revista Secuencia, el dossier «Los niños como actores sociales en la historia de América Latina (siglos XIX-XX)» en Trashumante y «Lectores, autores y voceadores: niños y prensa en América Latina (1890-1945)». El compartir ese interés común con otras historiadoras e historiadores latinoamericanos nos permitió crear, por ejemplo, la Red de Historia de las Infancias en América Latina en 2015.

J. A.: ¿Qué aportes hacen los estudios historiográficos de la infancia a la comprensión de las problemáticas de los niños y niñas en el tiempo presente?

S. S.: El principal aporte es demostrar fehacientemente que lo que pensamos sobre la infancia hoy como natural o universal, no lo es. El principal aporte de la historia al entendimiento de las infancias actuales es que nos permite combatir los esencialismos; ideas tan violentas como la de «inocencia infantil», que hemos repetido desde hace siglos y que seguimos asociando con una característica esencial de la infancia, y que, sin embargo, es usada para despojar a los niños y niñas de su independencia, de su autonomía, de su capacidad de comprensión de los fenómenos que los rodean. La asociación de niñez-inocencia proviene del romanticismo del siglo XVIII y ha sido utilizada para eliminar la agencia infantil. No podemos, dice el Estado chileno hoy, por ejemplo, hablarles a los niños de política, porque ellos no entienden nada; porque eso supone una suerte de «ideologización»; porque rompemos la «burbuja de inocencia» en donde les corresponde vivir, como si los niños no fueran sujetos políticos, cuerpos políticos. Eso no se debería poder sostener si uno respeta mínimamente la infancia o si uno estudia mínimamente la historia de los niños, en donde se ve su enorme capacidad de entender el 
mundo en el que viven. Diarios infantiles como los que hemos rescatado en Latinoamérica (el de Conxita Simarro, catalana refugiada en México luego de la guerra civil española o Francisca Márquez, niña chilena que escribe durante la dictadura) destronan la falsa idea de incapacidad de los niños de pensar políticamente que prima hoy sobre políticos, maestros y padres de familia.

La historia también permite mostrar, por ejemplo, cuánto hay de falso en las ideas sobre feminidad y masculinidad que se construyen sobre los cuerpos infantiles. Cómo estas ideas preconcebidas de lo que una niña o un niño hace «naturalmente» atentan contra la diversidad de infancias que se pueden vivir. La paradoja es muy fuerte, por ejemplo, cuando se siguen asociando ideas del género a partir de dicotomías asentadas en objetos de la cultura material carros-muñecas, colores azul-rosa o acciones acción-ternura. La historia muestra que el color rosa y el azul, por ejemplo, son imposiciones comerciales; que si observamos pinturas del siglo XVIII veremos que los niños visten de rosa y las niñas de azul o blanco, y que eso no se vinculaba necesariamente con identidades genéricas.

La historia del trabajo infantil, por ejemplo, nos permitiría entender —con mucha más profundidad y cuidado- los movimientos de niños trabajadores organizados en Latinoamérica. Porque desde hace siglos los niños nos están mostrando su capacidad de organizarse, de trabajar, de sacar adelante a sus familias. En muchos expedientes que yo revisé de niños detenidos por pequeñas infracciones en el siglo XX, las madres solicitan la libertad de sus hijos, no solo por amor, sino porque dependen de ellos económicamente.

Entonces, el estudio de la historia de las infancias ofrece herramientas para pensarlas no como categorías biológicas, psicológicas, absolutamente esencializadas a partir de conceptos hegemónicos, construidos casi siempre desde el mundo de los saberes científicos, casi siempre desde el norte global, sino como categorías en constante cambio, fluidas, posicionadas en contextos y lugares específicos. La historia da herramientas para relacionarnos con los niños y las niñas respetuosamente; para hablar con ellos y escuchar sus voces desde otro lugar que no sea la relación jerárquica y vertical que hemos establecido hasta ahora con ellos.

Desde una perspectiva menos optimista, la historia también nos sirve para ver las grandes permanencias, procesos de larga duración. Hoy, por ejemplo, me encuentro estudiando la historia del secuestro infantil en México y veo con tristeza cómo, a pesar del endurecimiento de las leyes y las sanciones, el problema persiste. Los niños y las niñas siguen siendo tratados como objetos; siguen siendo abusados, mercantilizados, transportados como cuerpos-cosas. Son problemas tan profundos, con raíces tan extensas, que no parecen fáciles de resolver en el corto plazo. 
J. A.: Algunos autores, desde la historia y la sociología, insisten en diferenciar la infancia (como construcción social) y los niños y las niñas (como sujetos históricos y actores sociales). ¿Es posible evidenciar esta diferencia epistemológica y ontológica en algunos estudios que hayas desarrollado?, ¿qué funciones cumplen el Estado, el saber científico, la literatura, las publicaciones periódicas, los medios de comunicación o el mercado en la configuración de representaciones dominantes sobre los niños y las niñas?

S. S.: En mis primeros trabajos planteaba esta diferenciación de forma un poco más categórica. Porque siempre ha sido más fácil - debido a la dificultad de encontrar fuentes para hacer historia de las infancias- estudiar las representaciones sobre los niños que sus propias experiencias. Tenemos entonces muchos estudios sobre la forma en que médicos, literatos, periodistas, fotógrafos, cineastas, juristas o maestros, por mencionar algunos, imaginaron la infancia o construyeron políticas o producciones culturales para ella. Pero no necesariamente hay oposición entre discursos y prácticas. Foucault justamente planteó cómo los discursos son prácticas también. No hay una línea en donde empiece uno y termine el otro. De tal forma, podemos observar que si el cine mexicano de la época de oro, por ejemplo, representó a los niños trabajadores como pequeños héroes de su tiempo, siempre emprendedores de sí en una especie de melodrama de su vida cotidiana, esas representaciones cinematográficas estaban dialogando con otros discursos y creando unos nuevos; incluso generaban formas de tratar y ver a los niños y partían de ciertas observaciones del comportamiento infantil.

Cuando se estudia a los niños trabajadores del pasado, estos nos muestran su enorme capacidad de tomar decisiones, de resolver problemas, de asumir riesgos y de calcular consecuencias. Los niños vendedores de la prensa, por ejemplo, tenían muy claro en qué esquinas podían vender mejor, debían decidir cuántos ejemplares pedir en el expendio de manera que no les sobraran, además asumían la gran responsabilidad que implicaba su salario para su familia y entonces calculaban cuánto gastar en el cine o en revistas para poder todavía llegar con algunos pesos a su casa. En los años treinta un gran número de familias de sectores populares estaban conformadas por la madre y sus hijos, y eran estos los que colaboraban en el sostenimiento familiar. De tal forma, la infancia de estos niños no cuadraba con las representaciones que hacían los adultos, que pretendían que todos los niños y las niñas se definieran en función de su dependencia, subordinación y falta de autonomía. Ni siquiera estos niños y niñas, generalmente de los sectores populares, encajaban en esas ideas que sostenían que la infancia ideal estaba alejada de las prácticas sexuales; todo lo contrario.

Todo esto nos invita a pensar cuántos estereotipos manejamos sobre la infancia, cuántas veces hemos repetido una idea unívoca de la infancia que responde a todos esos esencialismos 
que niegan la pluralidad de infancias que existen. La historia entonces nos permite dejar de hablar de infancia y comenzar a hablar de infancias.

J. A.: De acuerdo con las investigaciones realizadas sobre la infancia en momentos específicos de la historia de México, ¿cómo captar «la voz»o la experiencia de los niños y las niñas en las investigaciones historiográficas?

S. S.: Este ha sido el talón de Aquiles de los últimos años de avances historiográficos en temas de infancias. Las y los historiadores nos habíamos tenido que concentrar en lo que los adultos decían y hacían sobre los niños y las niñas. Sus miradas, sus observaciones, los términos que usaron para referirse a los niños, que daban cuenta de una relación vertical en donde las voces infantiles quedaban silenciadas, matizadas e, incluso, tergiversadas. No obstante, si uno mira con cuidado y tiene como objetivo recuperar las pequeñas voces - que a veces son tan solo murmullos, pero otros gritos estridentes- en muchas de esas fuentes construidas por los adultos, trabajadoras sociales, pedagogos, juristas, médicos, periodistas, en donde solo encontrábamos construcciones adultas, las voces infantiles llegan a ser tan potentes que es imposible no escucharlas. Ahí están los niños detenidos por los Tribunales de menores, explicando por qué se han robado una herramienta del taller artesanal, justificando estas acciones porque los maestros artesanos los han dejado sin pago; ahí están las niñas que trabajaron como servidoras domésticas y que en venganza a los maltratos huían con pertenencias de sus empleadoras o incluso llegaron a quemar las casas; ahí están las niñas que se quejan de haber denunciado un abuso sexual frente a un mundo masculino que les pidió una y otra vez repetir la misma narración. También ahí están los niños que a través de sus dibujos expresaban lo que entendían de obras de teatro pesadamente aleccionadoras, que los niños probaban como poco exitosas en términos de ideologización. Y están las niñas diaristas, esas niñas escritoras, muy observadoras de su presente, que se colocaron en el centro de la Historia para narrarla desde su punto de vista. En ellas vemos voces que desde lo más íntimo; analizan las circunstancias que les toca vivir y nos dejan testimonios de la vida infantil de otras épocas, de las formas en las que las niñas jugaban, se relacionaban con sus pares y con los adultos, construían sociabilidades, consumían libros y películas y se preocupaban por el amor y la amistad.

A estas alturas del camino ya no tenemos como pretexto el tema de las fuentes para no estudiar la vida de niños y niñas del pasado. Ya hay muchos avances historiográficos en nuestra región que nos muestran de qué manera como historiadores podemos construir fuentes; por ejemplo, a través de la entrevista, a través de la etnografía; pero también y, especialmente, a través de la creatividad, con las fuentes que están en los archivos, con las cartas que los niños escriben a los periódicos, con los archivos personales y familiares. 
J. A.: De acuerdo con lo que señalas en la introducción del libro Nuevas miradas a la historia de la infancia en América Latina: entre prácticas y representaciones sobre de la dificultad de encontrar fuentes historiográficas no adultocéntricas en los archivos, ¿qué valoración haces de los estudios narrativos y biográficos sobre la historia de la infancia?

S. S.: Hay textos que, desde la ficción, han retratado la vida de los niños en la historia; hay otros que lo hacen desde un posicionamiento autobiográfico, desde una construcción de la memoria de un adulto que busca dejar registro de ciertos aspectos de su niñez. Todo nos sirve para reconstruir la vida de los niños. Lo que he sostenido en mis trabajos es que debemos ser cuidadosos con las fuentes y establecer la operación primaria del trabajo del historiador, que es la crítica de esas fuentes: ¿quién la produjo?, ¿en qué momento?, ¿por qué?, ¿cuál fue su objetivo?, ¿qué quiere decir?, ¿qué dice y qué no dice?, ¿por qué lo dice? No es lo mismo el diario de una niña de once años que escribe desde la mesa que está bajo la ventana de la cocina de su familia o tapada por las cobijas de su cama para que nadie la vea, a las memorias de un pintor, un político o una médica, que escriben sobre su infancia una vez que esta ha terminado y en donde en el transcurrir de las décadas siguientes la memoria ha borrado ya muchas cosas y agrandado otras. He tratado de insistir en que debemos partir de la fuente que tenemos. ¿Tenemos las palabras que escribió un niño o una niña o las palabras de adultos que intentan recuperar lo que les queda de memoria sobre su infancia? Son dos cosas distintas. Las memorias y autobiografías tienen un lugar de enunciación del sujeto que como investigadores debemos explicitar. Además de los procesos de olvido, los autores toman decisiones sobre lo que quieren o no quieren publicar, sobre lo que quieren o no quieren que leamos de su vida o sobre la figura que quieren construir a la posteridad a través de esa escritura. Las niñas diaristas - hablo de niñas porque no han aparecido diarios de niños latinoamericanos todavía- a veces escriben para la posteridad, pero su escritura es un intento de comprenderse a sí mismas; su escritura es una lucha contra el olvido. Son conscientes de los embates del tiempo en la memoria y por eso narran a veces con lujo de detalles o de horarios lo que les ocurre. Lo narran desde la infancia, no desde la memoria de la infancia que los autobiógrafos elaboran ya muy lejos de la experiencia infantil con afán de publicar en una editorial, de limpiar su nombre, de explicar sus decisiones o dejar esbozada una imagen específica de ellos para los lectores.

J. A.: Desde hace algunos años, algunos investigadores de la infancia en la región, quizás bajo el influjo de perspectivas de la llamada historia cultural, han explorado fuentes como la fotografía, el cine, la literatura infantil y los juguetes, entre otros artefactos o representaciones culturales. ¿Qué valoración haces de estas apuestas investigativas? 
S. S.: Yo soy feliz cada vez que aparece una nueva investigación sobre la historia de los juguetes, la cultura material infantil o las diversas representaciones de la infancia en la historia de América Latina. Solo con todas esas contribuciones tendremos una más amplia perspectiva de cómo fueron las experiencias de las diversas infancias en la región. No solo eso, el estudio de fotografías o de cine, de la literatura o el teatro nos permiten advertir muchos de los estereotipos que han circulado en torno a los niños y niñas. Ahora tenemos estudios muy interesantes sobre la cultura material que rodeó a los niños y niñas desde las revistas infantiles, hasta la decoración de las habitaciones de los niños de clase media. Desde la historia de la educación, por ejemplo, se puede ver cómo el aula se va adaptando a los cuerpos de los pequeños. En el cruce entre la historia de la publicidad y la historia de las infancias podemos observar, especialmente en la segunda mitad del siglo XX, cómo crece exponencialmente el mercado de productos que se ofrecen para los niños y cómo esos productos expresan nítidamente un contexto histórico social determinado. No es fortuito que los niños de los años cuarenta recibieran anuncios publicitarios de ambulancias o metralletas de plástico, mientras que las niñas jugaban con pequeñas muñecas enfermeras que se anunciaban como objetos para «lograr la paz».

J. A.: ¿Qué recomendaciones haces sobre el estudio de la infancia en la historia reciente de América Latina y el Caribe, especialmente en sus relaciones con los fenómenos de neoliberalización, las nuevas formas de consumo cultural y la pantallización e hiperconexión de medios digitales en las sociedades contemporáneas?

S. S.: En Latinoamérica quienes más han trabajado la historia reciente de la infancia en la región han sido los grupos de Argentina, Brasil y Chile. Estos equipos se han enfocado especialmente en la historia de las infancias bajo el contexto de las dictaduras militares. Son trabajos fundamentales para comprender la relación entre la represión del Estado y la infancia. En Colombia la veta más potente en historia reciente de la infancia ha tenido que ver con los procesos de violencia que ha vivido el país. Tenemos algunos aportes también para la historia de Centroamérica y la participación de los niños en las guerrillas. Pero nos falta mucho por hacer. Pienso que, a pesar del crecimiento de este campo de estudio, de sus amplias posibilidades y las perspectivas que ofrece, todavía niñas y niños siguen siendo considerados por muchos historiadores como sujetos poco importantes, ajenos a las grandes decisiones políticas y económicas que parecen mover las macrohistorias. De tal modo, si bien las historiadoras e historiadores de la infancia hemos abierto caminos para pensar las infancias desde una perspectiva histórico cultural, temas como la relación entre infancias y neoliberalismo o las nuevas formas de comunicación masiva están todavía poco explorados, siendo campos que ofrecerían perspectivas importantes para entender cómo los niños han sido integrados, por ejemplo, a la sociedad de 
consumo. Desde las primeras décadas del siglo XX es posible identificar claramente la búsqueda de construir a los niños como pequeños consumidores. Se les integró muy temprano en la economía de mercado global; su imagen se utilizó como un poderoso gancho en anuncios publicitarios; se les incitó a visitar las tiendas y probar todo tipo de productos diseñados ya especialmente para ellos, para su tamaño, sus intereses y capacidades. El mercado infantil es de una importancia global fundamental en la actualidad. Tenemos también en América Latina ya varios trabajos que apuntan a historizar esa dimensión, pero es un campo abierto para nuevas y necesarias investigaciones. 